\title{
Design of 3-D Compound Tactons for Navigation Information Display
}

\author{
Zhen Jia ${ }^{1, a}$, Jianqing $\mathrm{Li}^{1, b}$ and Wanpei Geng ${ }^{1, \mathrm{c}}$
}

${ }^{1}$ School of Instrument Science and Engineering, Southeast University, Nanjing, 210096, China azjia1987@gmail.com, ${ }^{b}$ ljq@seu.edu.cn, ${ }^{c}$ tju_gwp@126.com

\section{Keywords: compound Tactons, navigation information, vibrotactile feedback}

\begin{abstract}
Different from transformational Tactons, compound Tactons are a sequence of two or more one-element Tactons. Previous studies have investigated the perception of transformational Tactons which encode two or more dimensions of information using various vibrotactile parameters. Little has been done on how to design compound Tactons to present non-visual information effectively. This paper investigates recognition rates for compound Tactons which encode multidimensional navigation information. Experimental results show that overall identification rate for three-parameter Tactons is $92.65 \%$ by using different vibrotactile parameters, suggesting compound Tactons are a promising method of conveying complex information. This study will guide designers to select suitable compound Tactons for use when navigating in a virtual or real urban environment.
\end{abstract}

\section{Introduction}

In today's modern and fast-paced society, one challenge is how to orient in unknown spaces. It is difficult to navigate in a strange place, even if there are fixed traffic signs. They cannot be seen once this place is very crowd, and are more unavailable to the blind people. Most navigation systems convey spatial information through the visual or audio channel, by which the information can nearly be accessed everywhere. But for the blind, they are neither hand-free nor an effective solution. Using visual and audio channels could always distract the users' attention, and the information may be overwhelmed due to sensory overload. In some cases, they can even be blocked, e.g. noise, darkness and frog. Moreover, these channels are more limited for the visually impaired. According to Wickens' multiple resource theory (MRT) [1], allocating information from one overloaded sense modality to another modality can reduce workload. Therefore, the alternative tactile channel can be employed to convey navigation information.

There have been a number of studies on using vibrotactile feedback for navigation. An intuitive way of displaying directional information was introduced by van Erp et al. [2], by generating vibrotactile stimuli on the torso to "point" in the moving direction. The ActiveBelt invented by Tsukada et al. [3] was a waist belt equipped with eight vibrators, producing vibrotactile stimuli around the waist to "point" into a horizontal direction. Distance presentation can be beneficial for spatial navigation, although direction information alone is adequate to reach a turn. McDaniel et al. [4] presented intimate, personal, and social interpersonal distances to the blind by using tactile rhythms. By using a tactile torso display, Pielot et al. [5] presented the location and spatial distance of several people in a fast-paced 3D multiplayer game. With respect to tactile icons ("Tactons"), the concept was first introduced by Brewster and Brown [6], including one-element, compound, inherited, and transformational Tactons. As structured vibrotactile messages, Tactons were later employed to provide the navigation feedback. Lin et al. [7] proposed a pedestrian navigation system of conveying navigation information to the sighted user by using Tactons. But their tactile navigation prototype is not hands-free. However, few studies have looked at the issue of using compound Tactons to present non-visual information.

In this paper, compound Tactons are first employed to convey multidimensional navigation information in the form of vibrotactile stimulus. The vibration wave propagation is explored to clarify the theoretical basis. A psychophysical experiment is performed to evaluate the effectiveness of multidimensional Tactons of communicating complex information. Experimental results indicate that compound Tactons have good performance in conveying complex navigation information. 


\section{Vibration Wave Propagation}

Two types of vibration motors are commonly used for vibrotactile feedback: pancake motors and cylindrical motors. Different from pancake motors, the eccentric mass of cylindrical motors rotates perpendicularly to the skin. To this end, cylindrical motors are more likely to generate intense sensations. In our study, the cylindrical motor encased in a plastic mold was selected to produce vibrotactile stimulus (see Fig. 1). The plastic encasement makes the motor more robust, and increases the contact area between the motor and the skin.

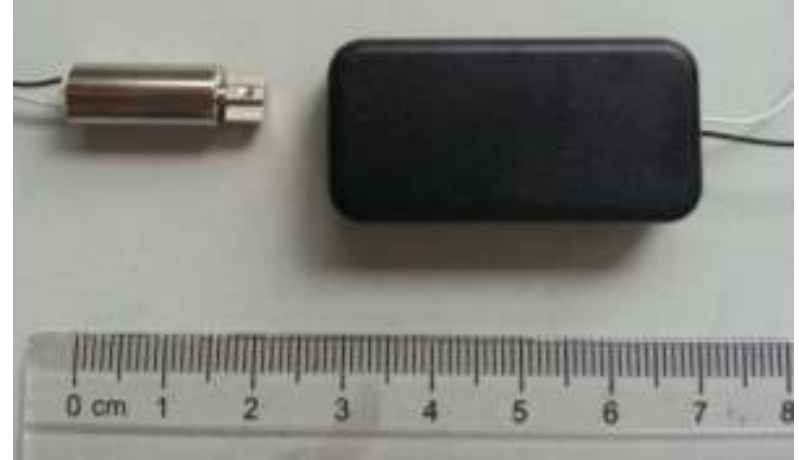

Figure 1. A cylindrical motor and a plastic encasement.

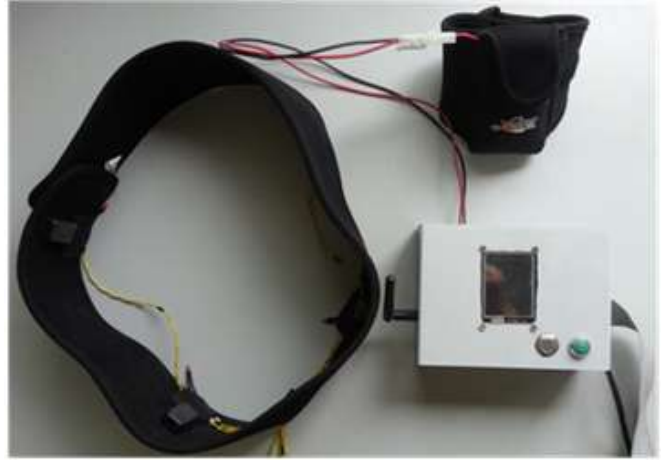

Figure 2. Prototype of wearable tactile system.

When the vibrator oscillates vertically on the skin, a longitudinal wave will travel downwards and a shear wave along the surface. Longitudinal and shear waves will combine at different angles. The wave equation for plane longitudinal waves propagating in the $x$-direction is

$$
\frac{\partial^{2} V_{x}}{\partial t^{2}}=c_{L}^{2} \frac{\partial^{2} V_{x}}{\partial x^{2}} \text {, with } c_{L}=\sqrt{\frac{\xi+2 \mu}{\rho}}
$$

where $V_{x}$ is the displacement of particles in the skin in the $x$-axis direction, $c_{L}$ the propagation or phase velocity, $\xi$ and $\mu$ called the Lame constants, and $\rho$ the density of the skin. The wave equation for plane shear waves is the same, except the propagation velocity $c_{S}$ :

$$
\frac{\partial^{2} V_{y}}{\partial t^{2}}=c_{S}{ }^{2} \frac{\partial^{2} V_{y}}{\partial x^{2}}, \text { with } c_{S}=\sqrt{\frac{\mu}{\rho}}
$$

The displacement of the particles is in the $y$-axis direction. The wave equation for a wave created by sinusoidal excitation and propagating in the positive $x$ direction is generally solved as

$$
V(t, x)=A \cdot e^{j(w t-k x)} \text {, with } k=\frac{2 \pi}{\lambda}=\frac{\omega}{c}
$$

where $A$ is the amplitude of the wave, $k$ the wave number, $\lambda$ the wavelength, and $c$ the propagation speed [8].

\section{Experiment Design}

Experimental Setup. The apparatus was consisted of navigation information acquiring module, kernel control module, navigation information display module, and vibrotactile feedback module, as well as power supply. The information acquiring unit was a NRF24LO1 wireless module, which can receive the transmitted navigation information wirelessly, and send it to the kernel control module; the kernel control module was a STM32VET6 controller, whose functions were decoding navigation information, and encoding tactile language in the form of vibrotactile stimuli; the information display module was a TFT-LCD screen; the vibrotactile feedback module included the vibrotactile driving circuit and a waist belt, of which four vibrating motors were attached to the inside of the belt, and the driving circuit enabled vibrators to produce vibrotactile stimulus. Besides the waist belt, a backpack was chosen to carry the experimental apparatus. The main controller and the battery were enclosed within a plastic box, which was positioned vertically in the backpack, with extended control lines connected to the belt. Fig. 2 presents the prototype of the entire tactile system. 
Design of 3-parameter Tactons. The tactile icons were structured to convey three pieces of information: the "Waypoint Type", "Distance" and "Heading Direction" of a hypothetical navigation message. The Waypoint Type could be "Crossing", "Destination" or "Landmark"; the Distance could be either "Far" or "Close"; the Heading Direction could be "Right", "Back", "Left" or "Front". This gave 24 different message types. Three salient parameters of vibrotactile stimulation were used to create the icons: stimulation direction, intensity variation and body location. Accordingly, twenty-four tactile patterns were designed by manipulating three parameters: direction (counterclockwise, simultaneous and clockwise), intensity variation (decreasing and increasing), and body location (navel, spine, the left and right sides). Figure 3 demonstrates the mappings between icon and message type.

To indicate what type of the next waypoint, the four vibrators on the tactile belt turned on and off in a different direction for $1 \mathrm{~s}$. Counterclockwise $(C C W)$ was chosen to represent crossing messages, simultaneous $(S T)$ destination messages, and clockwise $(C W)$ landmark messages. The parameters of frequency and amplitude are unsuitable to encode information, but frequency and amplitude can be considered as one parameter of intensity. Here, decreasing intensity was used to denote far messages, and increasing intensity described close messages. In this course, the vibrator located on the front of the belt is activated for $1 \mathrm{~s}$. Directions can be divided into the cardinal and ordinal directions, and body location is the best tactile parameter of conveying direction information. In our study, we encode four different levels of directions (right, back, left and front) on the waist. One vibrotactile pulse is triggered on the belt to guide the walking direction.

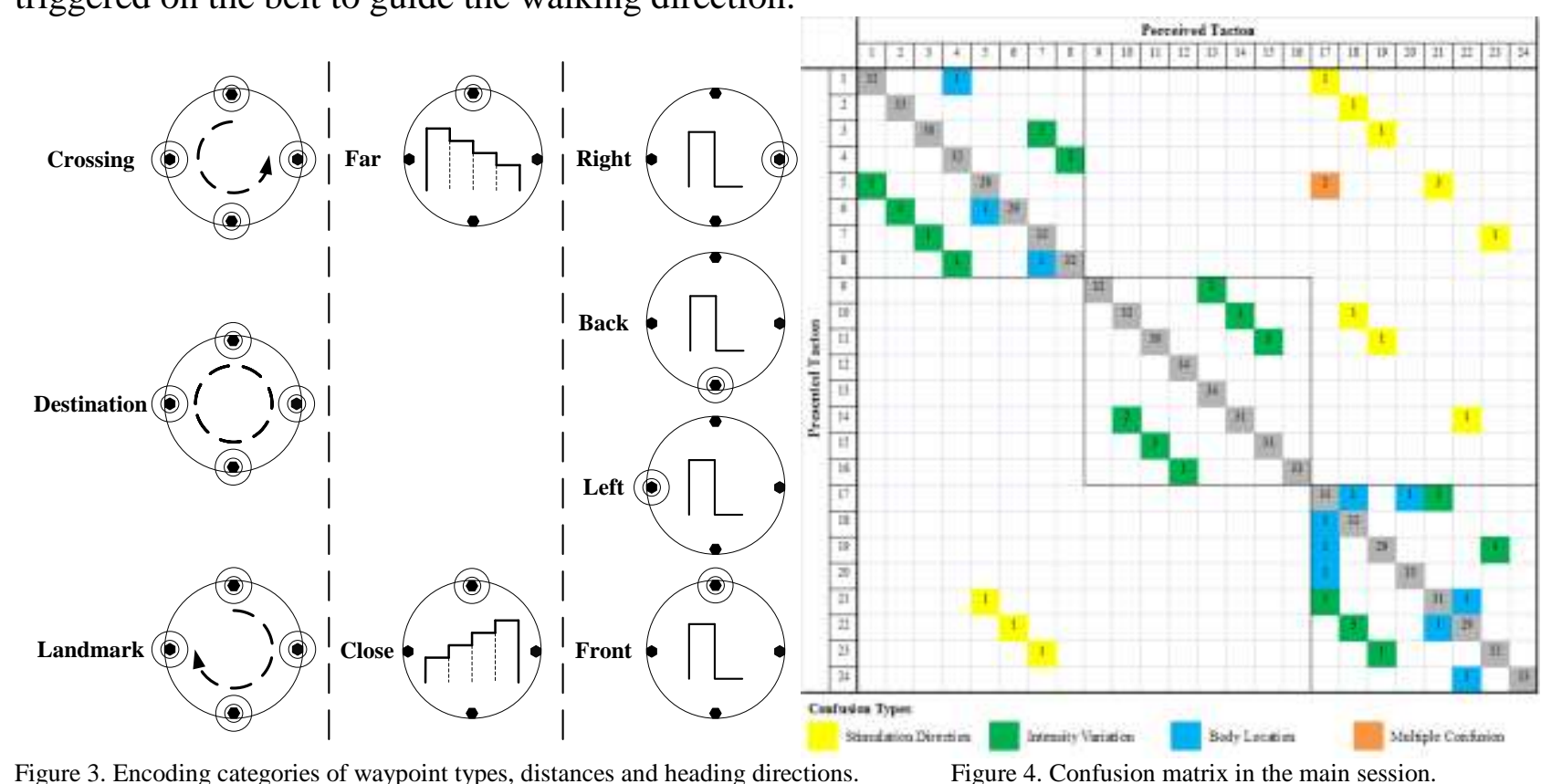

\section{Evaluation of 3-parameter Tactons}

Design and Procedure. Nine male and eight female adults participated in this experiment, aged from 23 to 29. They were all students from Southeast University. Before starting the experiment, subjects were trained to recognize the three-parameter Tactons. The concept of icons was introduced to them, and the mapping between vibrotactile parameters and attributes of the navigation messages were explained. Then they were allowed to perceive each icon several times in 15 minutes, by clicking the buttons on the training interface. During this time, they had to associate the icons with their meanings. After getting familiar with the icons, they took part in 24 tasks like those from the experiment itself as training in how to use the testing interface. Headphones were worn to block any noise from the vibrators.

All twenty-four icons were presented two times in a random order, resulting in 48 trials for each subject in the experiment. Therefore, there were a total of: 17 subjects $\times 3$ Stimulation Directions $\times 2$ 
Intensity Variations $\times 4$ Body Locations $\times 2$ presentations $=816$ trials. In each trial, one icon was repeated two times with a one second pause between repetitions. While the icon was being presented, the subjects had to identify all three attributes encoded in the icon, by selecting the corresponding radio buttons on the testing interface. They needed not to wait until the icon had been presented all two times before responding if they had already decided. When they had made their choice, they could submit their response so as to step into the next trial. All the experiment interfaces were built in a Visual C++ environment.

Result and Discussion. The confusion matrix of subjects' responses for the main session is shown in Figure 4. It can be derived that the overall mean accuracy for three-parameter tactile icons was $92.65 \%$. In this case, the information transfer $(I T)$ value was calculated to be 4.17 bits, corresponding to 17.97 discriminable icon types [9]. In addition, the confusion matrix for each parameter is illustrated in Figure 5. The mean accuracy for the three vibrotactile parameters was $98.16 \%$ for Stimulation Direction, 95.59\% for Intensity Variation, and 98.65\% for Body Location.

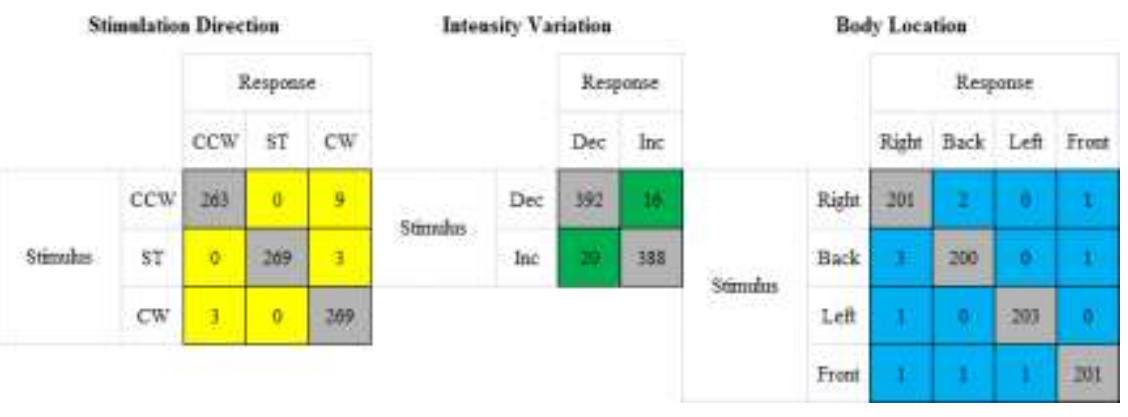

Figure 5. Confusion matrix for each parameter (errors of multiple confusions are not included).

The data for all measures violated the assumption of a normal distribution, following Shapiro-Wilk tests. Therefore non-parametric analyses were carried out. Effects of Stimulation Direction and Body Location were analyzed using a Friedman's test (non-parametric one-way ANOVA equivalent) while all pairwise comparisons (including for the effect of Intensity Variation) were conducted using Wilcoxon signed-rank tests. Friedman's analysis found no effect of Stimulation Direction on the number of correct responses $\left(\chi^{2}(2)=3.250, \mathrm{p}=0.197\right)$, with mean recognition rates of $96.69 \%$, $98.90 \%$ and $98.90 \%$ for the $C C W, S T$ and $C W$ respectively. There was also no significant effect of Body Location on correct response counts $\left(\chi^{2}(3)=1.531, \mathrm{p}=0.675\right)$. Mean recognition rates were 98.53\%, 98.04\%, 99.51\% and 98.53\% for Right, Back, Left and Front respectively. A Wilcoxon test showed no significant effect of Intensity Variation on correct response numbers $(\mathrm{Z}=-0.298, \mathrm{p}=$ 0.765). Mean recognition rates were $96.08 \%$ for Decreasing and $95.1 \%$ for Increasing. Figure 6 presents mean identification rates of all three-parameter tactile icon types.

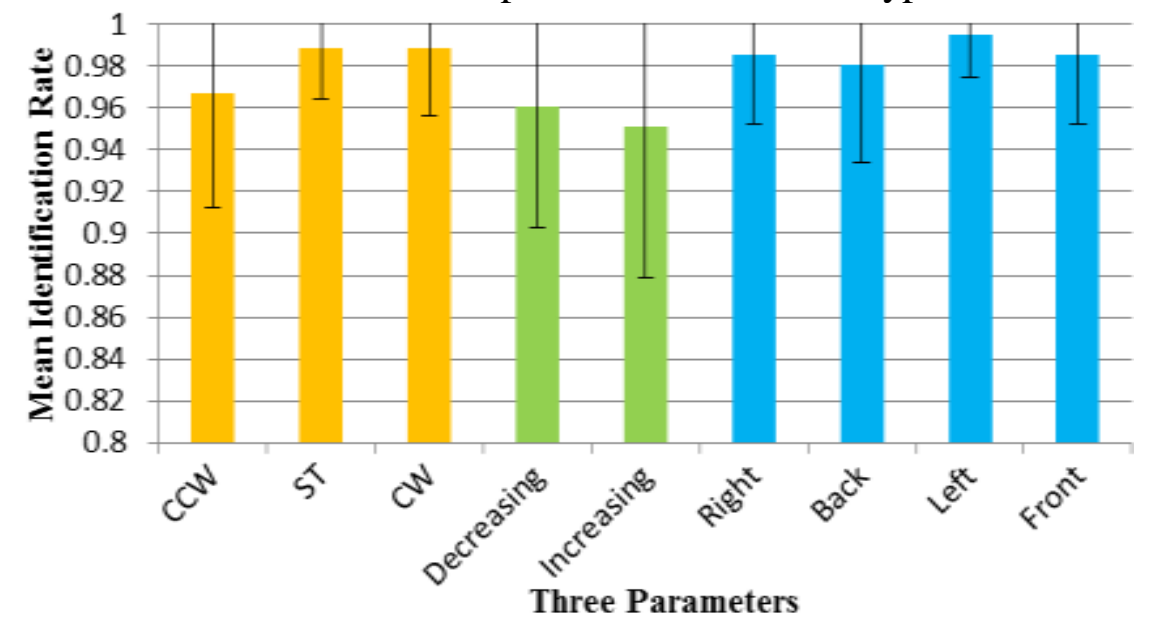

Figure 6. Mean Identification Rates for all icon types: stimulation direction, intensity variation, body location.

Note that the mean identification rate for three-parameter compound Tactons is very high, with $92.65 \%$ accuracy in identifying three pieces of navigation information. However, this figure is higher 
than the $80.56 \%$ identification rate for three-parameter transformational Tactons, even when Brown et al. reduced the levels of roughness parameter from three to two [10]. Since the results are promising, future work should address the slightly more error-prone parameter of Intensity Variation. Of the 60 errors, 36 confused the Intensity Variation of the icon, resulting in $95.59 \%$ accuracy for that parameter. Due to fewer errors, Stimulation Direction and Body Location both had a higher accuracy of $98.16 \%$ and $98.65 \%$ respectively. Meanwhile, the amount of information transmitted is large for three-parameter Tactons, at 4.17 bits corresponding to 17.97 icons. It means almost 18 icons can be identified correctly of all 24 tactile icons. Therefore, it is a promising way to convey complex information by using compound Tactons.

\section{Conclusion}

This paper has conducted the first study of compound three-parameter Tacton design and identification. Experiment results are encouraging, as it is easy to identify three pieces of navigation information from vibrotactile icons. A necessary next step is testing identification while the user is walking in a virtual or real urban environment, as these initial results show compound Tactons to be similarly effective in conveying multidimensional information as Earcons and transformational Tactons. However, the heading directions are limited to right, back, left and front in this study, while a pedestrian has more choices of orientation in the environment that needs more accuracy. In our future work, adding another four ordinal directions around the waist will be considered. With the purpose of reducing navigation errors, four-parameter tactile icons will be structured by following the design principle of three-parameter Tactons. Another piece of information could be, for instance, the "Route Attribute" of the navigation message. The alternative tactile parameter may be thermal change direction, as it is proved to be particularly salient [10]. But it needs to be investigated whether confusion will be produced when presenting four different tactile stimuli sequentially.

\section{Acknowledgement}

This work is supported by the Doctoral Program of Higher Education of China (20130092110060), and the Natural Science Foundation of Jiangsu Province (BK2012560).

\section{References}

[1] C. D. Wickens, S. Hutchins, and T. Carolan. Human Factors (2013), 55(2): pp. 461-70.

[2] L. R. Elliott, J. van Erp, and M. Duistermaat. IEEE Transactions on Haptics (2010), 3(2): pp. $78-87$.

[3] K. Tsukada, and M. Yasumura. In Proceedings of UbiComp 2004, pp. 384-399.

[4] T. McDaniel, S. Kirshna, and D. Colbry. In Proceedings of CHI 2009, pp. 4669-4674.

[5] M. Pielot, O. Krull, and S. Boll. In Proceedings of CHI 2010, pp. 1-10.

[6] E. Hoggan, R. Raisamo, and S. A. Brewster. In Proceedings of ACM ICMI 2009, pp. 327-334.

[7] M. Lin, Y. Cheng, and W. Yu. In Proceedings of the 5th Nordic Conference on Human-Computer interaction: Building Bridges (2008), 358: pp. 507-510.

[8] J.W. Streefkerk, W. K. Vos, and N.J. Smets. In Proceedings of the Human Factors and Ergonomics Society (2012), 56: pp. 277-281.

[9] H. Z. Tan, C. M. Reed, and N. I. Durlach. IEEE Transactions on Haptics (2010), 3: pp. 98-108.

[10] G. Wilson, S. Brewster, and M. Halvey. In Proceedings of MobileHCI 2012, pp. 309-312. 